\title{
NUMERICAL STUDY OF PURGE AND SECONDARY FLOWS IN A LOW PRESSURE TURBINE
}

\author{
Jiahuan, Cui \\ CFD Laboratory, Department of Engineering \\ University of Cambridge, UK \\ jc763@cam.ac.uk \\ Paul, Tucker \\ CFD Laboratory, Department of Engineering \\ University of Cambridge, UK \\ pgt23@cam.ac.uk
}

September 14, 2016

TURBO-16-1142 Cui 


\section{abstract}

The secondary flow increases the loss and changes the flow incidence in the downstream blade row. To prevent hot gases from entering disk cavities, purge flows are injected into the mainstream in a real aero-engine. The interaction between purge flows and the mainstream usually induces aerodynamic losses. The endwall loss is also affected by shedding wakes and secondary flow from upstream rows. Using a series of eddy-resolving simulations, this paper aims to improve the understanding of the interaction between purge flows, incoming secondary flows along with shedding wakes and mainstream flows on the endwall within a stator passage.

It is found that for a blade with an aspect ratio of 2.2, a purge flow with a $1 \%$ leakage rate increases loss generation within the blade passage by around 10\%. The incoming wakes and secondary flows increase the loss generation further by around 20\%. The purge flow pushes the passage vortex further away from the endwall and increases the exit flow angle deviation. However, the maximum exit flow angle deviation is reduced after introducing incoming wakes and secondary flows. The loss generation rate is calculated using the mean flow kinetic energy equation. Two regions with high loss generation rate are identified within the blade passage: the corner region and the region where passage vortex interacts with the boundary layer on the suction surface. Loss generation rate increases dramatically after the separated boundary layer transitions. Since the endwall flow energizes the boundary layer and triggers

TURBO-16-1142 Cui 
earlier transition on the suction surface, the loss generation rate close to the endwall at the trailing edge is suppressed.

\section{INTRODUCTION}

The trend with commercial aero-engines is to increase by-pass ratio, especially through the introduction of gear boxes. This means the diameter and aspect ratio of Low-Pressure Turbines (LPTs) continue to decrease. Currently, the secondary loss accounts for around $30 \%$ of the overall loss in an axial turbine. With a decreased aspect ratio, higher secondary loss is expected.

Secondary loss, or endwall loss, arises from the interaction between the pitchwise pressure gradient and the inlet boundary layer on the endwall. Due to the high loss associated with secondary flow, a great deal of studies have been dedicated to this topic. Some basic understanding of secondary flow features and the associated loss mechanism have been established. Figure 1 shows a sketch of endwall flow features and some possible disturbances from the upstream rotor and purge flow. When the incoming endwall boundary layer approaches the blade leading edge, it rolls up to form a vortex, i.e., the horseshoe vortex. Two legs (pressure leg and suction leg) of the horseshoe vortex move into the adjacent blade passages. Under the effect of cross flow, the pressure leg develops to the passage vortex, which is the dominant flow structure in the endwall region. Since the incoming boundary layer is separated and washed away, a new laminar boundary layer is created 


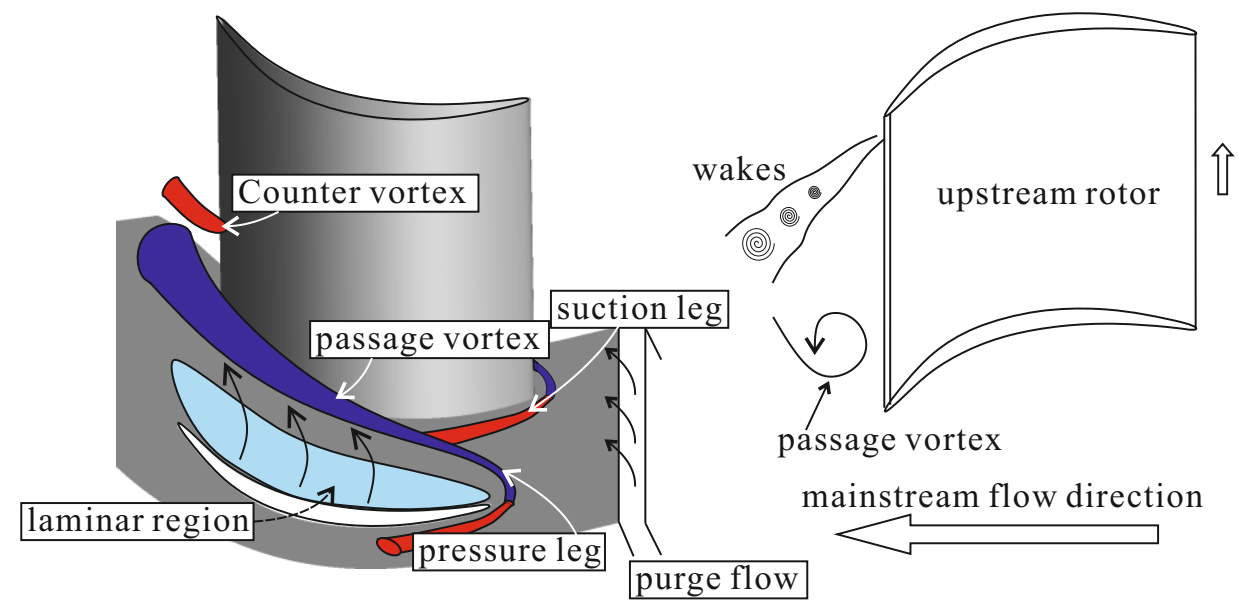

Figure 1: Sketch of endwall flow and possible external disturbances from upstream rotor.

downstream of the pressure leg on the endwall [1]. A shear layer is formed at the trailing edge between the fluid on the suction side and the fluid on the pressure side. The spanwise velocity component on the suction side is much larger than on the pressure side. This results in a vortex with an opposite sign to the passage vortex. This is the counter vortex, or the trailing shed vortex.

So far, most studies on the endwall flow are either experimental or lowfidelity (RANS) modelling. Detailed experimental measurements in the region downstream of the Trailing Edge (TE) have been reported [2]. However, due to the limitation of accessibility no measurements were taken within the blade passage.

Low-fidelity modelling has been used extensively to understand endwall flows $[3,4,5]$. RANS simulations can roughly capture the endwall flow features. 
However, the complex transition scenario on the endwall compromises the accuracy of RANS simulations. The RANS equations generally assume the state of the boundary layer on the endwall is fully turbulent. In reality, the state of inlet endwall boundary layer is potentially transitional rather than fully turbulent. In addition, a new laminar boundary layer covers much of the endwall downstream of the pressure leg of the horseshoe vortex. These all lead to an over prediction of the endwall loss by RANS.

In real aero-engines, secondary flows are under constant external forcing. The purge flow from inter-blade-row gaps increases the boundary layer thickness and distorts the inflow angle close to the endwall. Depending on the leakage flow rate, a separated flow region can appear downstream of the inter-blade row gap. The intrinsic instability of a separated shear layer increases the turbulent kinetic energy and further enhances the mean flow energy dissipation. The purge flow has been found to increase the secondary loss and penetration depth of endwall flows [4].

Apart from the purge flow, shedding wakes accompanied by the secondary flow from the upstream blade rows interact with the downstream endwall flow. Steurer [6] experimentally studied the effects of unsteady wakes on endwall loss. In their experiments, upstream moving bars were used to generate wakes. The effects of secondary flow from the upstream rotor were not represented. The strong secondary flow from an upstream rotor can distort the inflow angle and secondary flow pattern in the downstream blade row [7].

The current study focuses on the combined effects of the purge flow and 


\begin{tabular}{ll} 
Table 1: Specification of T106A cascade \\
\hline Reynolds number $(R e)$ & $1.6 \times 10^{5}$ \\
Chord $(C)$ & $198 \mathrm{~mm}$ \\
Axial chord $\left(C_{x}\right)$ & $170 \mathrm{~mm}$ \\
Pitch & $158 \mathrm{~mm}$ \\
Span $(h)$ & $375 \mathrm{~mm}$ \\
Suction surface length $\left(S_{0}\right)$ & $265 \mathrm{~mm}$ \\
Inlet angle $(\alpha)$ & $-39.7^{\circ} 1$ \\
Design exit flow angle $(\beta)$ & $63.2^{\circ}$ \\
Aspect ratio $\left(h / C_{x}\right)$ & 2.2 \\
\hline
\end{tabular}

unsteady secondary flow with wakes from an upstream rotor on endwall losses. To current authors' knowledge, no high-fidelity numerical studies have been reported on this topic and there is limited detailed experimental data.

\section{COMPUTATIONAL FRAMEWORK}

T106A profile is used in the current study. Table 1 shows the specification of T106A cascade. In total, six test cases are studied and they are summarized in Table 2. Of these, purge and WSP are purge flow cases focusing on the effects of the hub leakage on the endwall flow; while $L B L$ and $W S$ are the cases with the same inflow boundary conditions without purge flows injected into the mainstream. The purge (no blade) is designed to study the isolated effect of the hub leakage and $L B L$ (full span) is to investigate the effect of

\footnotetext{
${ }^{1}$ It is worth noting that the flow angle measured in the experiment was $-37.7^{\circ}$. As explained by Sandberg et al. [8], the uncertainty of the inflow angle measurement can be high. The authors have tested a range of solvers with $-39.7^{\circ}$ inflow angle and they all show encouraging agreement. The $2^{\circ}$ difference was necessary to give the correct pressure distribution. We note other works [8] need more substantial incidence change.
} 


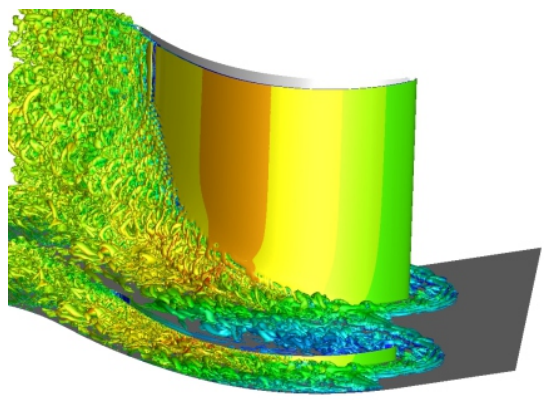

LBL

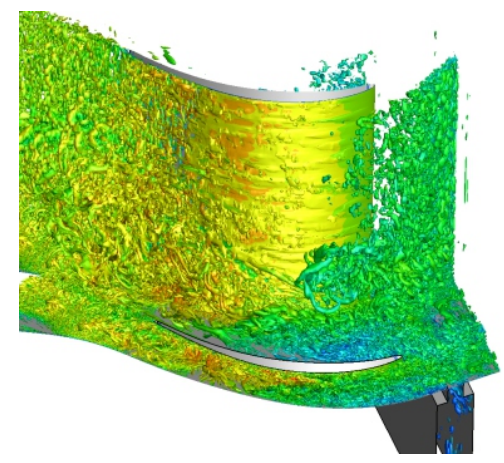

WSP

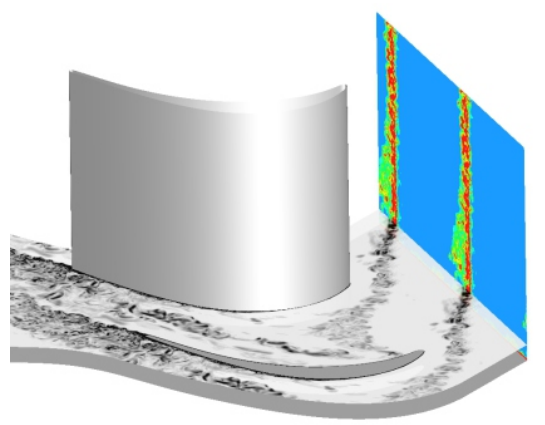

WS

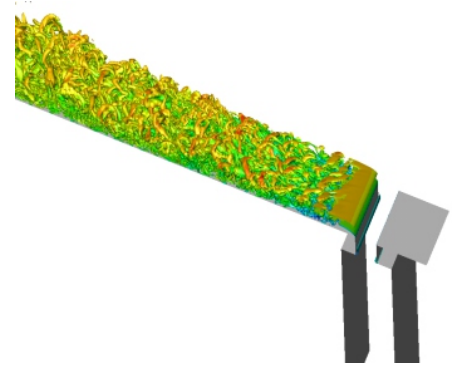

Purge (no blade)
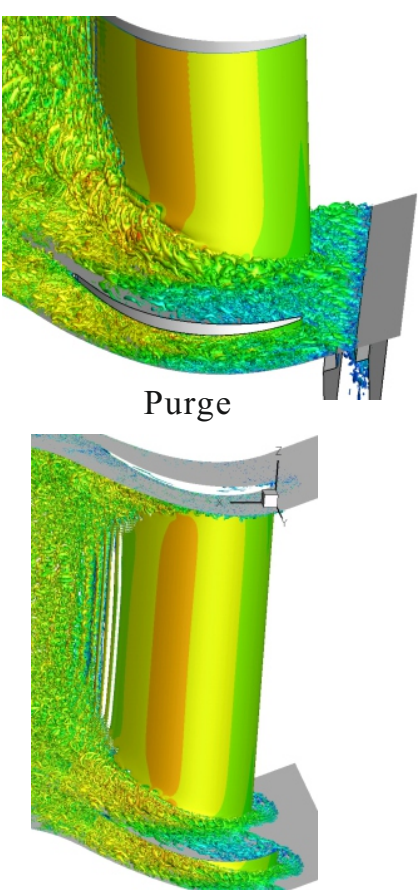

LBL (full span)

Figure 2: Snapshots of instantaneous flow fields for all the test cases (LBL, WS, Purge, WSP, Purge with no blade and LBL (full span)). The vorticity magnitude countours are ploted at the inlet and on the endwall for WS case. The isosurfaces of $Q$-criterion are plotted for other cases. 
Table 2: Test cases

\begin{tabular}{lcc}
\hline Inlet flow condition & Test case acronym & Span resolved \\
\hline Laminar Boundary Layer & LBL & $50 \% h$ \\
Wakes and Secondary flows & WS & $50 \% h$ \\
LBL with purge flows & Purge & $50 \% h$ \\
WS with purge flows & WSP & $50 \% h$ \\
Purge flow without blade & Purge (no blade) & $50 \% h$ \\
LBL & LBL(full span) & $100 \% h$ \\
\hline
\end{tabular}

the simplification of resolving $50 \%$ span. Snapshots for all the cases are given in figure 2 .

No endwall flow measurements were taken for the T106A cascade. However, the experiment by de la Blanco [9] was carried out in the same low speed wind tunnel in the Whittle laboratory at the University of Cambridge. To minimize uncertainty in the inflow boundary condition, the quasi-laminar velocity profile measured by de la Blanco, as shown in figure 3, is specified at the inlet for the $L B L$, purge, purge (no blade) and LBL (full span) cases.

The incoming wakes and secondary flows from the upstream rotor is generated from a precursor simulation. The instantaneous velocity data at the trailing edge $\left(x / C_{x}=1.01\right)$ in the LBL case is extracted. To match the Reynolds number of the other cases, the velocities are scaled down as

$$
u_{j} / U_{i n}=u_{j} / \sqrt{u_{i}^{\text {mid }} u_{i}^{\text {mid }}}
$$

where the velocities on the right hand side are at the trailing edge in the LBL case. Supscripts mid denotes the quantities at the midspan. The subscprit in 


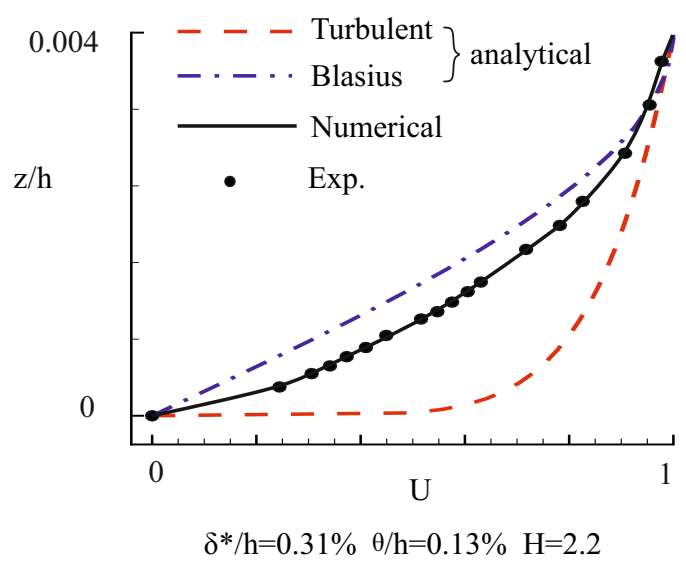

Figure 3: Mean boundary layer velocity profile on the endwall at the inlet compared with the measurements [9].

denotes the quantities at the inlet. After scaling, a method based on velocity triangles[10] was used to rotate the velocity vector to ensure the same pressure distribution at the midspan as the other cases. The upstream-rotor moving velocity $\left(=0.96 U_{i n}\right)$ was chosen to represent the condition of a repeating stage at $50 \%$ reaction.

The computational domain and boundary conditions are shown in figure 4. To make the computation feasible, a pair of periodic boundaries is imposed in pitchwise direction so that the computational domain only covers a single blade passage. An O-mesh is used to resolve the boundary layer around the blade; while the rest of the blade passage is discretized by an H-mesh. To reduce the mesh skewness near the trailing edge, an unstructured mesh block is placed downstream of the blade. A buffer zone, located $0.3 C_{x}$ downstream of the trailing edge, is used to avoid any reflections from the outlet boundary. Similar to Xia, et al.[11], a higher smoothing coefficient is used in the buffer 


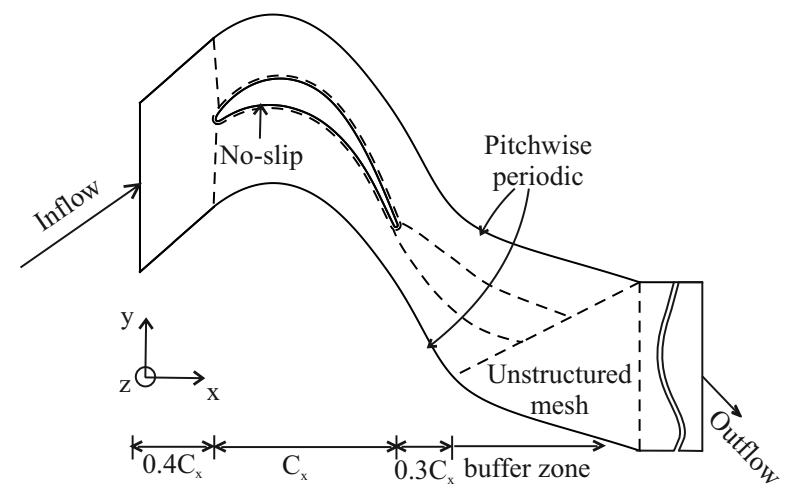

Figure 4: $x-y$ plane view of the computational domain with boundary conditions.

zone. To keep the simulation free of any excessive dispersion, a small coefficient is applied in the rest of the domain. No sub-grid scale model is used.

For purge flow cases, a cavity is placed at $0.15 C_{x}$ upstream of the leading edge. The cavity geometry is the same as the one used in the experiments by de la Blanco [12]. Figure 5 shows the geometry and boundary conditions for the purge flow cases. The inflow velocity at the lower boundary of the cavity is set to introduce a leakage flow rate of $1 \%$.

The total number of mesh cells used for the simulations in this study is approximately 40 million $(1158 \times 216 \times 160)$. A grid independence study, doubling number of cells from around 40 million to 85 million, has been performed. All the parameters of interest are found to be insensitive to the mesh resolution. (See appendix A for more details.) Figure 6 shows the refined mesh resolution in wall units on the blade surface. In the region of primary interest $(0<z / h<30 \%)$ the wall units are within the range of 


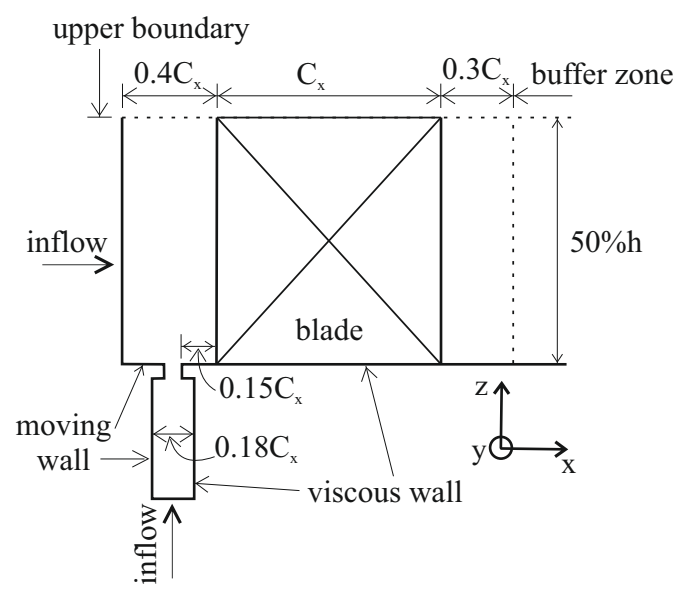

Figure 5: $y-z$ view of the computational domain with boundary conditions for the cases with purge flows.

$\Delta y^{+}<2, \Delta x^{+}<30$ and $\Delta z^{+}<40$. Figure 7 shows the mesh resolution in Kolmogorov units $(\chi)$. The Kolmogorov length scale can be calculated by $\chi=\left(\nu^{3} / \epsilon\right)^{1 / 4}$ where $\nu$ is the kinematic viscosity and $\epsilon$ is the dissipation of turbulent kinetic energy. It is assumed that the local turbulent kinetic energy is in the equilibrium state. Therefore, the dissipation rate is approximated by the production term $\epsilon \approx P_{t}=-\left(\overline{u_{i}^{\prime} u_{j}^{\prime}}\right) \partial \overline{u_{i}} / \partial x_{j}$. The mesh size is calculated by taking the cubic root of the cell volume. It can be seen from figure 7 that the mesh resolution in Kolmogorov units is $<25$. This mesh resolution is of the same range as the large eddy simulation for the endwall flow within a compressor cascade presented by You et al. [13].

In the WSP case, around 30 mesh points at the inlet are used to resolve half wake-width. This is similar to the mesh resolution used by Wissink and Rodi [14]. To resolve the purge flow, around 100 mesh points are used in 
$x$-axial direction in the cavity.

Similar to the strategy adopted by You et al [15], half of the span is resolved and an inviscid wall boundary condition is placed at 50\% span. This simplification is justified by the fact that the endwall flow only penetrates to approximately $30 \%$ span. The effects of the inviscid wall boundary condition on the flow at the midspan is discussed in the Results and Discussion section.

The Rolls-Royce in-house solver - HYDRA is used for all the test cases. HYDRA is a second-order edge-based finite volume solver. It has been heavily modified for eddy-resolving simulations. The Rogers-Kwak scheme (artificial compressible method) [16] is implemented. This improves the performance for low-Mach number flows. The current authors have reported successful work focusing on the flow at the midspan using the same solver [17].

\section{RESULTS AND DISCUSSION}

\section{The effects of inviscid wall boundary at the midspan.}

Figure 8 shows the $Q$-criterion isosurface to visualize the vortical structure of flow on the suction surface and endwall. The boundary layer on the suction surface at the midspan is separated near the trialling edge, where Kelvin-Helmholtz (KH) roll-ups are formed. The separated boundary layer transitions and then reattaches before the trailing edge. The loss generation rate within the separated boundary layer after transition is relatively high. This is discussed in the loss generation rate subsection. 

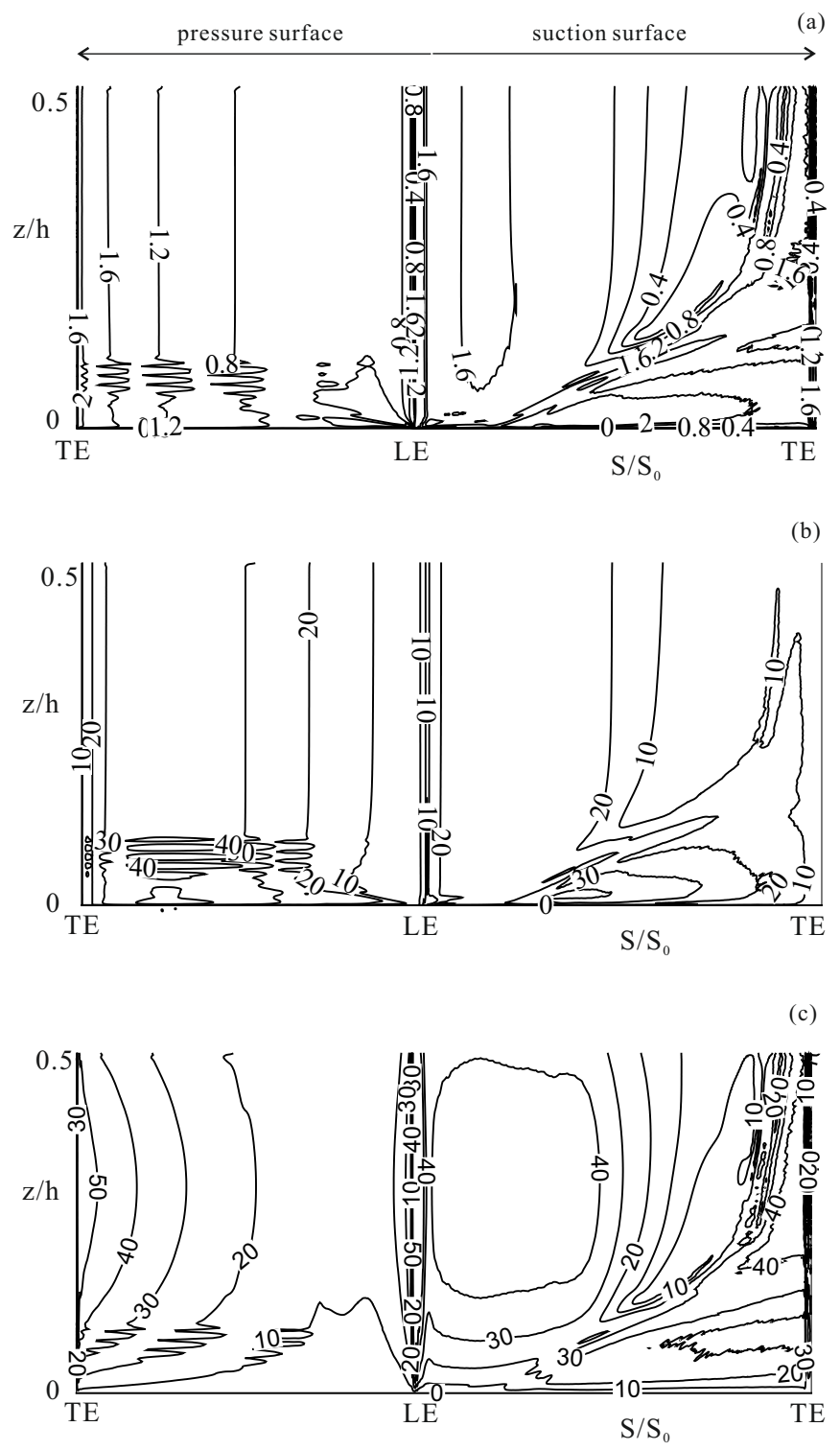

Figure 6: Mesh resolution in wall units on the blade surface. (a): $\Delta y^{+}$isoline, (b): $\Delta x^{+}$isoline, (c): $\Delta z^{+}$isoline. 


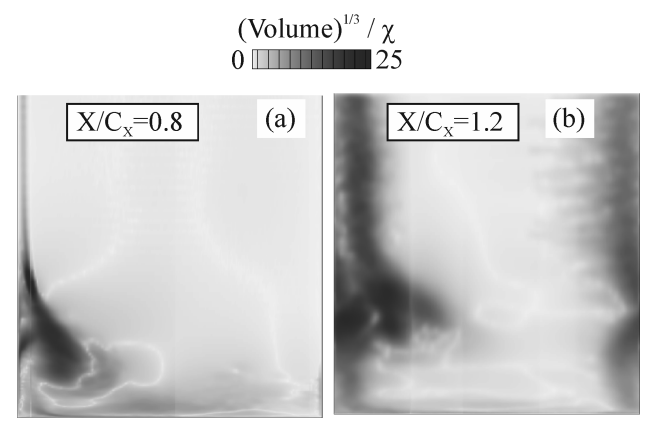

Figure 7: Mesh resolution in Kolmogorov units ( $\chi$ ) at (a) $x / C_{x}=0.8$ and (b) $x / C_{x}=1.2$.
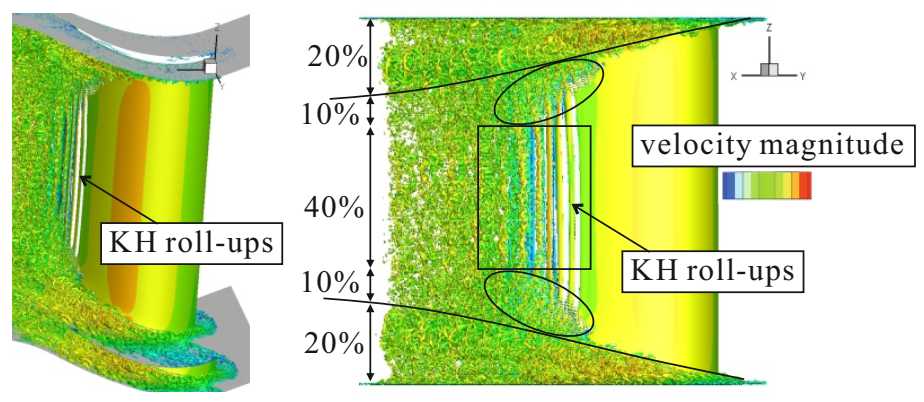

Figure 8: A snapshot of the $Q$-criterion isosurface $(Q=1000)$ contoured by velocity magnitude for the LBL (full span) case.

The passage vortex, which is the dominant endwall flow structure, penetrates to around $20 \%$ span at the trailing edge. The interaction between the passage vortex and the separated boundary layer on the suction surface affects another $10 \%$ span at the trailing edge. As a result, the quasi-2D flow covers around $40 \%$ span. It is worth noting that the aspect ratio of the T106A cascade is 2.2, which is smaller than the normal aspect ratio of LPTs in commercial aero-engines. Hence, the span affected by the endwall flow in real engines is less than in the current study. 
The influence of the simplification of resolving half span is shown in figure 9. The figure shows three sets of parameters at the midspan: (a) the pressure distribution on the blade surface, (b) the boundary layer velocity profiles on the suction surface near the trailing edge, and (c) the boundary layer integral parameters. These parameters are calculated at $45 \%$ of the span, where the measurements were taken. The available measurements [18] are also plotted as symbols in the figures.

A limited effect of resolving the half span is observed. A potential explanation for the difference between the results is that the midspan boundary condition in the half span case could be reflective. This marginally increases local turbulence levels and thus blockage. The latter would also result in the improved agreement with the measurements. Though marginal differences in velocity profiles and boundary layer integral parameters can be observed, these differences are relatively small and away from the endwall. Hence, it is believed that they do not impact on the conclusions made in this study.

Endwall flow features. Figure 10 shows the $Q$-criterion isosurface of the time-averaged flow solution. As the endwall boundary layer approaches the leading edge, it experiences an adverse-pressure gradient. A 3D separation bubble is formed. Due to the pitchwise pressure gradient (pointing from pressure to suction surface), the low momentum flow within the 3D separation bubble moves towards the suction surface. As this low momentum fluid meets the suction surface, it starts to interact with the boundary layer on 

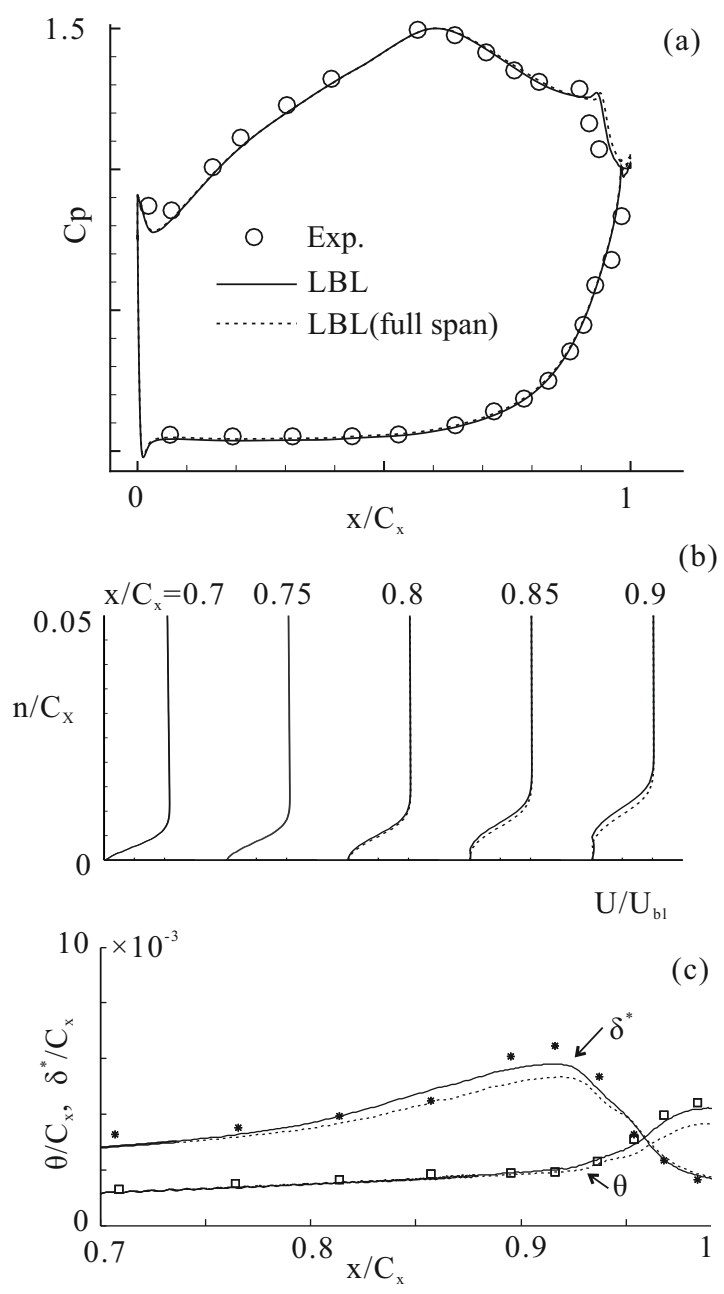

Figure 9: The parameters at the midspan for the half and full span resolved cases compared with available measurements: (a) pressure distribution on the blade, (b) boundary layer velocity profile on the suction surface near the trailing edge and (c) boundary layer integral parameters on the suction surface. 


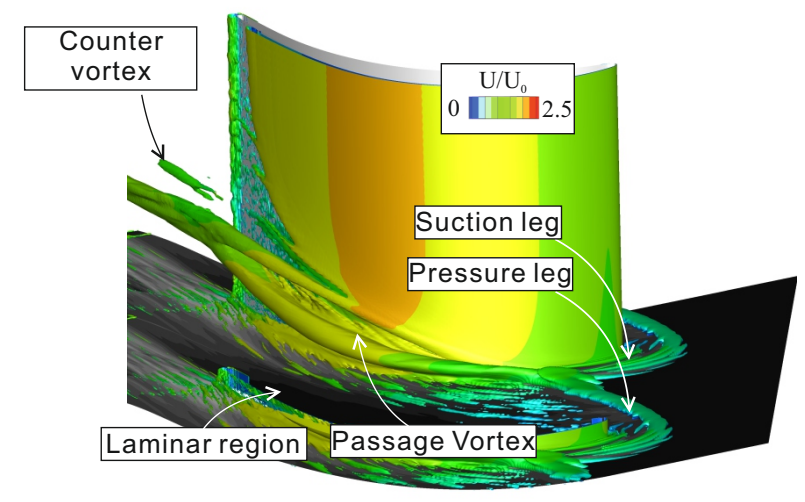

Figure 10: $Q$ isosurface of time-averaged flow solution of LBL case.

the suction surface ${ }^{2}$. This is the starting point of the passage vortex, which develops to be the dominant flow structure within aft portion of the blade passage. From the above analysis, the passage vortex originates from the momentum deficit of the endwall boundary layer. The momentum thickness of the endwall boundary layer is one of the major factors determining the size of the passage vortex.

Apart from the momentum thickness, the swirl angle ${ }^{3}$ close to the endwall also affects the endwall flow. The swirl angle determines how easily the endwall boundary layer is being turned towards the suction surface. A smaller swirl angle needs less turning, and thus the endwall flow interacts earlier with the boundary layer on the suction surface. As a result, more loss can be generated.

\footnotetext{
${ }^{2}$ This interaction increases the loss generation rate significantly (see loss generation rate subsection).

${ }^{3}$ Flow angle in tangential direction (or pitchwise direction in a linear cascade).
} 
Velocity and swirl angle upstream of the leading edge. Figure 11(a) shows the endwall boundary layer velocity profile and (b) the swirl angle at $x / C_{x}=-0.1^{4}$ upstream of the leading edge. From the figure, it can be seen that the incoming secondary flow from the upstream rotor representation distorts the velocity profile from $0.1 h-0.2 h$. The velocity increases by $\approx 5 \%$ at $0.11 h$, while it decreases by $\approx 2.5 \%$ at $0.14 h$.

Since the velocity profiles in figure 11(a) are extracted downstream of the purging slot, the impact of the purge flow is clearly visible. The momentum of the flow from the cavity is lower than that in the mainstream. This results in a decreased velocity magnitude from $0-0.01 h$. However, the purge flow also acts as a blockage. The mainstream flow is pushed away from the endwall, which essentially reduces the passage area and increases the velocity from $0.02 h-0.1 h$. This leads to a reduced loss coefficient observed at the exit from $0-0.1 h$ (see loss at the exit subsection).

In frame (b), the severe deviation of swirl angle caused by the different inflows is observed in two regions: $0-0.03 h$ and $0.1 h-0.2 h$. The incoming secondary flows (in WS case) increase the swirl angle in the region $0-0.03 h$, while a decreased swirl angle is observed in the region $0.1 h-0.2 h$. Apart from the incoming secondary flow, the region $0-0.03 h$ is also under the influence of the purge flow. The purge flow reduces the velocity magnitude and tangential velocity component. Hence, the swirl angle in the region $0-0.03 h$ is significantly reduced. This is shown by the velocity triangle II in

\footnotetext{
${ }^{4}$ The inset diagram shows the extracting location relative to the purging slot.
} 

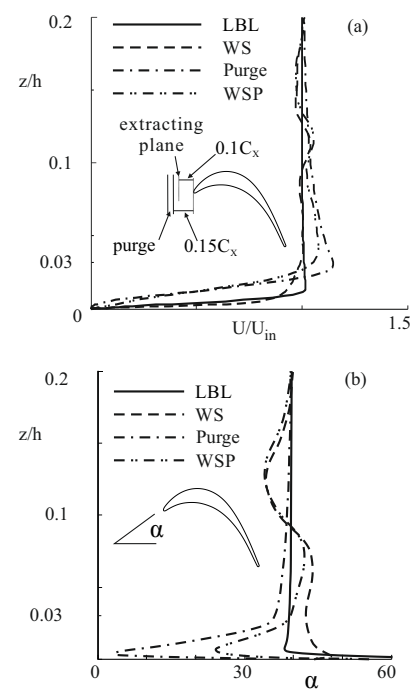

Figure 11: Pitchwise averaged profiles upstream of the leading edge: (a) dimensionless boundary layer velocity profile and (b) swirl angle.

figure 12. Because of the counter effect ${ }^{5}$ of the incoming secondary flow and purge flow in the region $0-0.03 h$, the swirl angle is less deviated in WSP case compared to the case with only the purge flow (velocity triangle III in figure 12).

Loss accumulation within the blade passage. Figure 13 shows the total pressure loss development in the $x$ direction. The local total pressure loss coefficient is calculated by

$$
C_{\text {loss }}=\left(P_{0 i n}-P_{0}\right) /\left(P_{0 i n}-P_{\text {exit }}\right)
$$

where $P_{0}$ is the local total pressure, $P_{0 i n}$ is the total pressure at the midspan upstream of the leading edge $\left(x / C_{x}=-0.1\right)$ and $P_{\text {exit }}$ is the static pressure

\footnotetext{
${ }^{5}$ The purge flow reduces the angle and the incoming secondary flow increases the angle.
} 


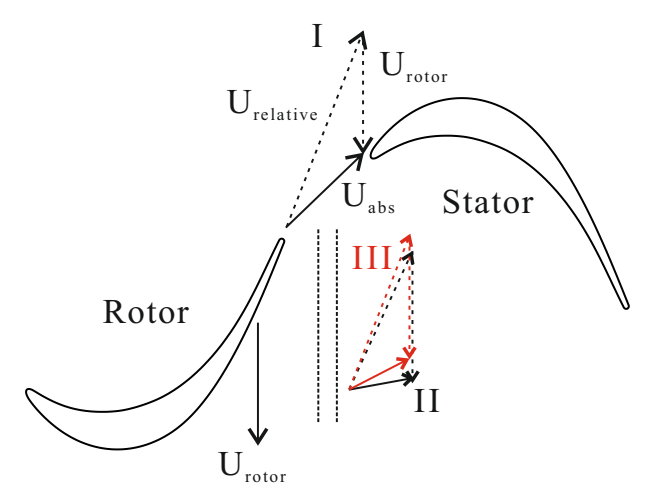

Figure 12: Velocity triangles for flows at the midspan (triangle I), close to the endwall after the purging slot without incoming secondary flow (triangle II) and with incoming secondary flows (triangle III).

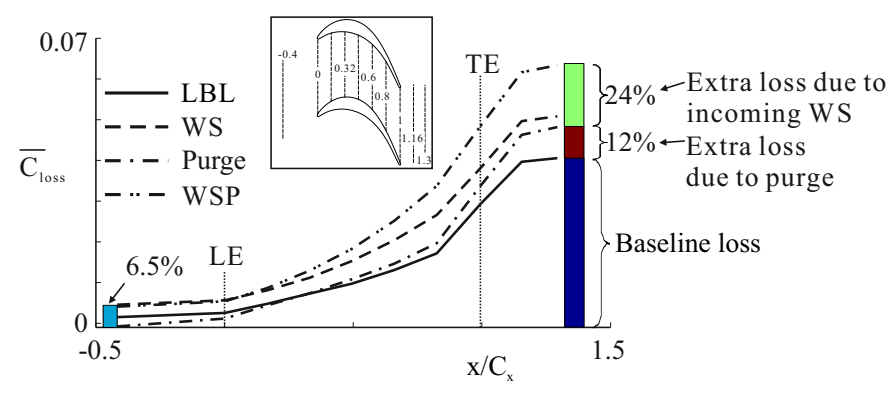

Figure 13: Mass-averaged total pressure loss coefficient.

at the exit $\left(x / C_{x}=1.3\right)$. This local total pressure loss coefficient is massaveraged over each of the 10 extracting plans as shown in the inset in figure 13. From the figure, approximately $36 \%$ more loss is observed at the exit for the WSP case compared to the LBL case, of which $6.5 \%$ is due to the velocity deficit of the incoming wakes and secondary flows at the inlet. That means around $30 \%$ more loss is generated within the blade passage due to the purge flow and incoming wakes and secondary flows.

To understand where the additional loss comes from in the WSP case, the 

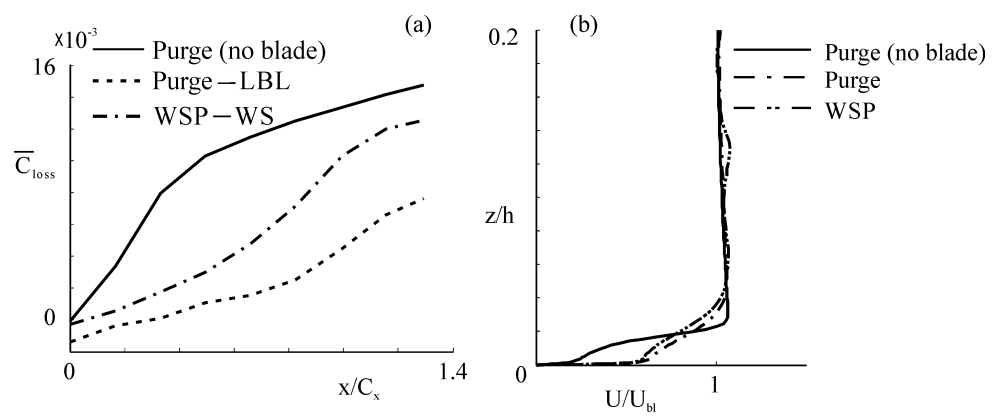

Figure 14: The interaction between endwall flows and purge flows: (a) loss from purge (no blade) case compared with the loss generated due to purge flows in the cases with blade, and (b) pitchwise averaged endwall boundary layer velocity profile at $x / C_{x}=0.16$.

following extra losses are examined separately: (1) loss due to the purge flow, and (2) loss due to the incoming wakes and secondary flow.

Extra loss due to purge flows. Since the purge flow is injected near the leading edge, a strong interaction between the purge flow and endwall flow is expected. This interaction generates an extra $12 \%$ loss within the blade passage (see figure 13). To understand the interaction between the purge flow and endwall flow, the loss from the purge (no blade) case is compared to the loss calculated by subtracting the loss in the LBL case from the loss in the purge case, see figure 14(a). Note that the loss in the purge (no blade) case is not affected by endwall flow. Since no blade is placed in the domain, there is no interaction between purge flow and endwall flow. Interestingly, the loss from the purge (no blade) case is almost twice as high as the loss difference between the purge and LBL cases. This suggests that the purge-endwall flow interaction can suppress loss generation. 
To understand why the purge-endwall flow interaction reduces loss generation, the endwall boundary layer velocity profiles at $x / C_{x}=0.16$ are plotted in figure 14(b). A stronger shear layer ${ }^{6}$ is observed in the purge (no blade) cases due to the separated boundary layer. In the purge case the separated boundary layer is suppressed by the favourable pressure gradient at the front portion of the blade passage. Since the shear layer is the origin of loss generation, stronger shear layer in the purge (no blade) case generates more loss compared to the purge case.

Extra loss due to incoming wakes and secondary flow. The loss due to the purge flow under the disturbances from incoming wakes and secondary flows is calculated by subtracting loss of the WS case from that of the WSP case. This loss is higher than the loss generated by purge flows in the cases without incoming wakes and secondary flows. The disturbances within the wakes and secondary flows enhance the mixing and thus increase the loss especially at the aft portion of the blade passage. However, the loss generated by purge flows in the cases with incoming wakes and secondary flows is not as high as the loss in the purge (no blade) case. The boundary layer is still being accelerated under the favourable pressure gradient in the front portion of the blade passage. As a result, the boundary layer velocity profile at $x / C_{x}=0.16$ is similar to that in the purge case.

Loss at the exit. Figure 15(a) shows the loss variation along the spanwise direction at the exit $\left(x / C_{x}=1.3\right)$. Three loss cores are identified in all of the

\footnotetext{
${ }^{6}$ In other words, larger velocity gradient.
} 

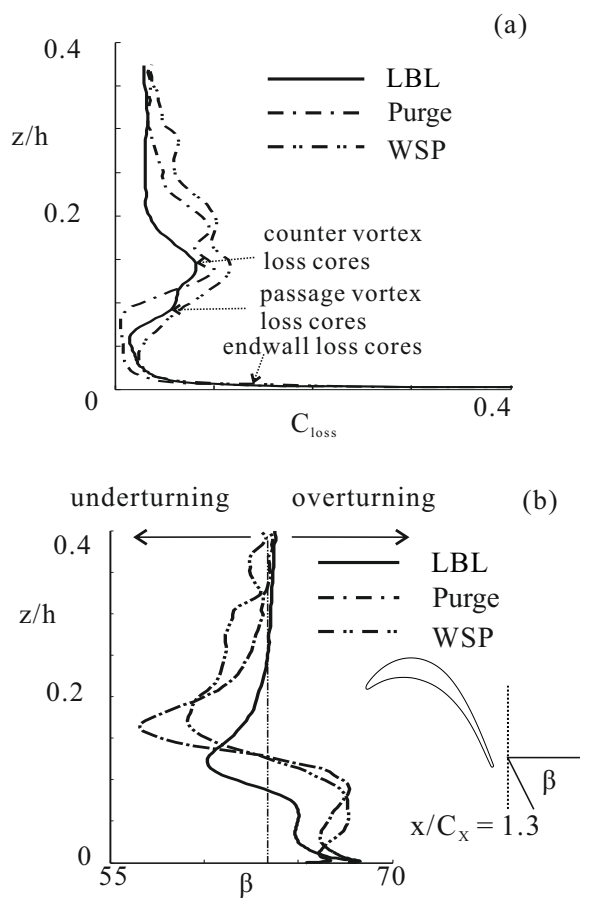

Figure 15: Pitchwise-averaged (a) loss and (b) velocity angle at the exit $\left(x / C_{x}=1.3\right)$.

cases. These are the endwall loss core, passage vortex loss core, and counter vortex loss core ${ }^{7}$. The highest loss is located within the boundary layer on the endwall (endwall loss core). A lower loss region between the endwall loss core and passage vortex loss core is observed for all the cases.

Except for the purge case, the loss within the region between endwall loss core and passage loss core is roughly the same as the loss at the midspan. A lower than midspan loss is observed for the purge case in this region. As discussed for figure 11, the velocity upstream of the leading edge close to the

\footnotetext{
${ }^{7}$ The loss cores in figure 15(a) are labelled only for LBL case.
} 
endwall in the purge case is higher in the region from $0.02 h-0.1 h$ than that at the midspan. This eventually leads to a smaller loss at the exit.

The passage vortex and counter vortex loss cores in the purge and WSP cases move further away from the endwall than in the LBL case. The purge flow also increases the loss of the passage vortex core significantly. As a result, the loss of the passage vortex core is at the same magnitude as that of the counter vortex core in the purge case. This is unlike in the LBL case where the passage vortex loss core is smaller than the counter vortex loss core.

Velocity angle deviation at the exit. The effects of the purge flow on strengthening the secondary flow can also be observed by the exit velocity angle, which is shown in figure 15(b). Compared to the LBL case, the overturning and underturning are both strengthened by the purge flow. The incoming wakes and secondary flows reduce the peak deviation of the underturning, while no obvious effects on overturning is observed.

The effect of the incoming secondary flow on smoothing out the velocity angle deviation can also be shown by the contours of the exit angle in figure 16 . A strong angle deviation is identified in the purge case, while this deviation is diffused by the disturbances from incoming wakes and secondary flows. Loss Generation Rate. The total pressure loss coefficient quantifies the loss generated between the inlet and the location where the coefficient is calculated. However, it cannot tell how fast the loss is generated at the questionable location. Denton [19] quantifies loss by entropy generation. In this way, local loss generation rate can be calculated by the entropy generation 


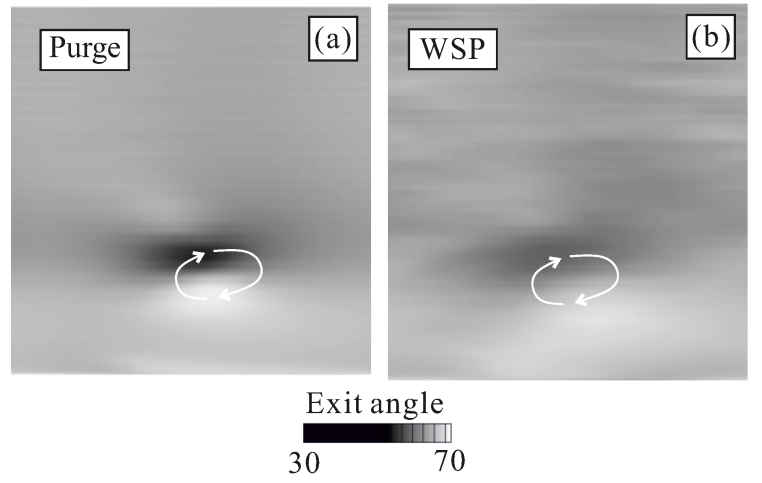

Figure 16: Exit angle contours for (a) Purge case and (b) WSP case.

rate, which is the sum of the following two components [20]

$$
\begin{gathered}
S_{v i s}^{\prime \prime \prime}=\frac{1}{T} \tau_{i j} \frac{\partial u_{i}}{\partial x_{j}} \\
S_{\text {therm }}^{\prime \prime \prime}=\frac{k_{e f f}}{T^{2}}\left(\frac{\partial T}{\partial x_{j}}\right)^{2}
\end{gathered}
$$

where $S_{v i s}^{i \prime \prime}$ is the entropy generation rate per unit volume due to viscous friction, and $S_{\text {therm }}^{\prime \prime \prime}$ is the generation due to heat transfer. Note, $T$ is the temperature, $\tau_{i j}$ is the shear stress tensor and $k_{e f f}$ is the effective heat conductivity.

Since the current study focuses on incompressible flows, mean flow kinetic energy equation [2] is used here. 


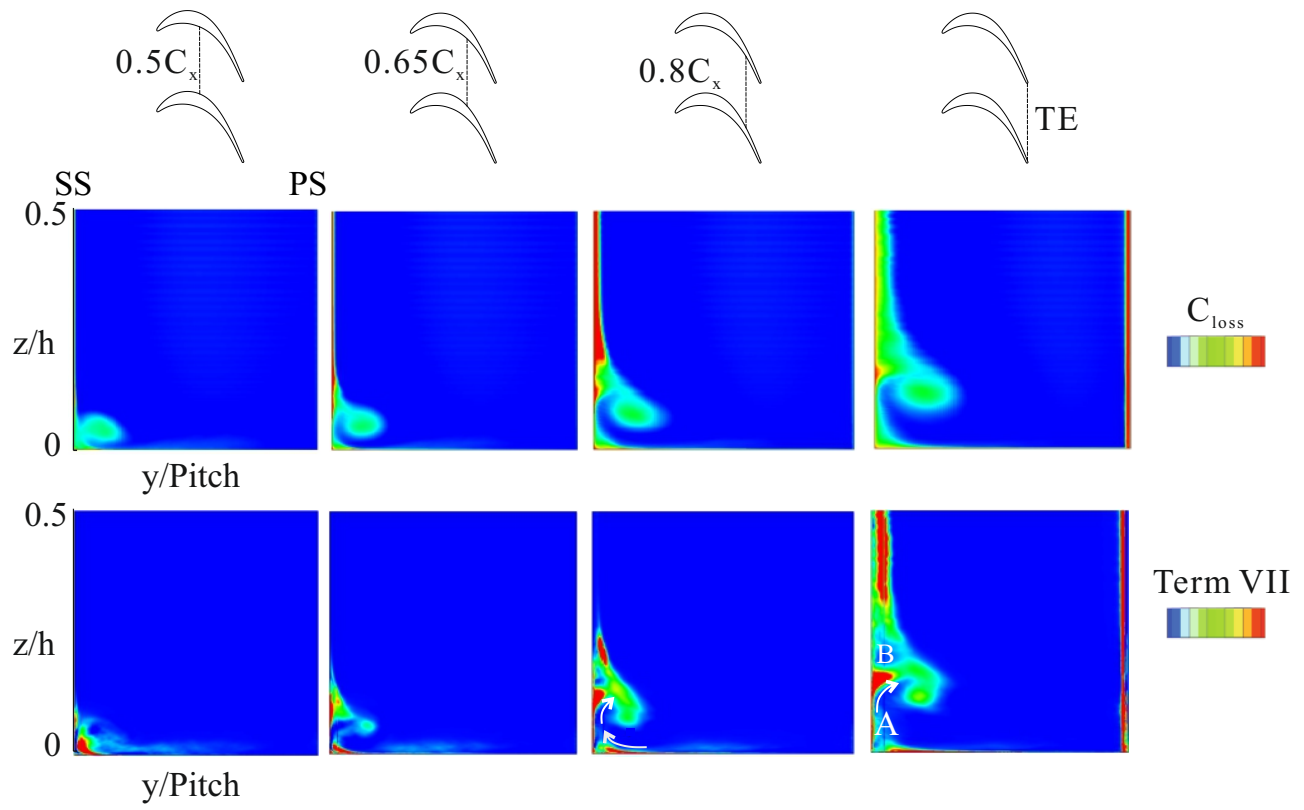

Figure 17: Total pressure loss and loss generation rate of term VII in equation 5.

$$
\underbrace{\frac{\partial E}{\partial t}}_{\mathrm{I}}+\underbrace{\bar{u}_{j} \frac{\partial E}{\partial x_{j}}}_{\mathrm{II}}=\frac{\partial}{\partial x_{j}} \underbrace{-\bar{u}_{j} \frac{\bar{p}}{\rho}}_{\mathrm{III}}+\underbrace{2 \nu \bar{u}_{j} \bar{s}_{i j}}_{\mathrm{IV}}-\underbrace{\left.\overline{u_{i}^{\prime} u_{j}^{\prime}} \bar{u}_{i}\right)}_{\mathrm{V}}-\underbrace{2 \nu \bar{s}_{i j} \bar{s}_{i j}}_{\mathrm{VI}}+\underbrace{\overline{u_{i}^{\prime} u_{j}^{\prime}} \bar{s}_{i j}}_{\mathrm{VII}}
$$

$E=\frac{1}{2} \bar{u}_{i} \bar{u}_{i}$ is the mean flow kinetic energy. $\bar{s}_{i j}$ is the rate of strain tensor. ${ }^{8}$ No backscatter is considered. It is assumed that all the turbulence kinetic energy is eventually dissipated to heat. The terms in the equation 5 are:

(I) the rate of change of mean kinetic energy;

(II) the rate of convective transport of mean kinetic energy;

$$
{ }^{8} \bar{s}_{i j}=\frac{1}{2}\left(\frac{\partial \bar{u}_{i}}{\partial x_{j}}+\frac{\partial \bar{u}_{j}}{\partial x_{i}}\right)
$$


(III) the rate of convective transport of static pressure;

(IV) the rate of mean kinetic energy transport by viscous stresses;

(V) the rate of mean kinetic energy transport by turbulence stresses;

(VI) the rate of viscous dissipation by mean velocity gradients and

(VII) the deformation work by turbulence stresses. It is also the rate at which turbulence is produced.

The terms II - V cannot generate or consume energy. They just redistribute it. Therefore, the dissipation of the mean flow energy, or the production of total pressure loss, is only due to two terms. They are term VI: the rate of viscous dissipation by mean velocity gradients, and term VII: the deformation work by turbulence stresses.

Figure 17 shows the local total pressure loss and loss generation rate from term VII at four different axial locations within the blade passage in the purge case ${ }^{9}$. The endwall boundary layer close to the suction surface experiences favourable pressure gradient on the front portion of the blade passage. Even though the suction side of horseshoe vortex travels near the suction surface, the interaction between the endwall flow and the boundary layer on the suction surface is not strong. This interaction starts to appear after $x / C_{x}=0.5$, where the passage vortex moves close to the suction surface and the pressure gradient on the suction surface becomes adverse.

As shown in figure 17 at $x / C_{x}=0.5$, the passage vortex loss core meets

\footnotetext{
${ }^{9}$ The loss generation rate of term VI is mainly from boundary layers where mean flow velocity gradient is high. Due to its relatively simpler mechanism, the contour of term VI is not given here. More details about this term can be found in Cui et al. [21].
} 
the suction surface. The loss generation rate at the corner between the suction surface and endwall is high. As the passage vortex moves downstream to $x / C_{x}=0.65$, the passage vortex loss core is washed up towards the midspan by the secondary flow. At this axial location, the boundary layer on the suction has been disturbed and a high loss generation region is observed on the suction surface.

At $x / C_{x}=0.8$, the boundary layer at the midspan has separated (see figure 9) ${ }^{10}$. The midspan loss on the suction surface starts to grow dramatically. Since the separated boundary layer has not transitioned yet, the loss generation rate at the midspan is still relatively low. At this axial location, three regions of high loss generation rate are observed: the corner region, the region next to the passage vortex, and the region above the passage vortex. The high loss generation rate in the corner region and the region next to the passage vortex is due to the washing-up of the endwall boundary layer and the boundary layer on the suction surface, respectively. For the region above the passage vortex, the high loss generation rate is caused by the early transition of the separated boundary layer. As mentioned above, the boundary layer has already separated at this axial location. The disturbance from the endwall flow triggers earlier transition and thus generates higher loss than the boundary layer at the midspan.

The separated boundary layer on the suction surface at the midspan

\footnotetext{
${ }^{10}$ More details about the boundary layer behaviour at the midspan can be found in Cui et al. [17].
}

TURBO-16-1142 Cui 
transitions before the trialling edge. As shown in figure 17 at the trailing edge $^{11}$, this transition thickens the boundary layer and thus increases the loss on the suction surface significantly. Since the boundary layer becomes turbulent after the transition, the loss generation rate increases to a high level within the boundary layer on the suction surface. Close to the endwall, the high loss generation region next to the passage vortex becomes larger and moves further away from the endwall compared to the ones upstream. Interestingly, there are two regions on the suction surface labelled as A and $\mathrm{B}$ where the loss generation rate is relatively low compared to that at the midspan. The lower loss generation rate in region $\mathrm{A}$ is due to the fact that the boundary layer within that region never transitions. This is the same region observed in figure $15(\mathrm{a})$ from $0.02 h-0.1 h$ where the loss is even smaller than that at the midspan. For the region B, the earlier transition of the separated boundary layer (as shown at $x / C_{x}=0.8$ ) suppresses the growth of the separation bubble. Hence, the turbulence generated within region B is much lower than at the midspan. Overall, the endwall flow generates higher loss. However, at the trailing edge, there are two regions where the local loss generation rate is reduced by the endwall flow.

\footnotetext{
${ }^{11}$ The last column of loss and loss generation rate contours.
} 


\section{CONCLUSIONS}

A range of eddy-resolving simulations for purge and secondary flows under the disturbances from upstream rotor have been carried out in this study. Key results are:

(1) Purge flows and disturbances from upstream rotors can significantly increase the loss within the stator especially for a low aspect ratio blade.

(2) Purge flows strengthen the endwall flow by increasing the penetration depth of the passage vortex and the deviation of the exit flow angle.

(3) The favourable pressure gradient close to the suction surface on the front portion of the blade passage can reduce the loss from the purge flow.

(4) Two regions with extremely high loss generation rate are identified: the corner region between the suction surface and the endwall and the region where endwall flow interacts with the boundary layer on the suction surface.

\section{ACKNOWLEDGEMENTS}

The authors would like to acknowledge Cambridge Overseas Trust for supporting this project. The permission from Rolls-Royce to publish this work is also gratefully acknowledged. Thanks are also due to Dr. Nagabhushana Rao Vadlamani, Dr. Rob Watson, Dr. Ifthekar Naqavi, and Mr. Ashley Scillitoe for their valuable discussions and inputs. 


\title{
NOMENCLATURE
}

\author{
Acronyms \\ KH Kelvin-Helmholtz \\ LE Leading Edge \\ LPT Low Pressure Turbine \\ PS Pressure Side \\ RANS Reynolds-Average Navier-Stokes \\ SS Suction Side \\ TE Trailing Edge \\ Greek symbols \\ $\alpha \quad$ Flow angle ahead of leading edge \\ $\beta \quad$ Exit flow angle \\ $\theta \quad$ Boundary layer momentum thickness \\ $\delta^{*} \quad$ Boundary layer displacement thickness \\ $\chi \quad$ Kolmogorov length scale \\ $\epsilon \quad$ Dissipation rate of turbulent kinetic energy \\ $\tau_{i j} \quad$ Shear stress tensor \\ Roman symbols
}


$C_{\text {loss }}$ Loss coefficient

$C_{x} \quad$ Axial chord

$C_{p} \quad$ Pressure coefficient $\left(P_{0 i n}-P\right) /\left(P_{0 i n}-P_{T E}\right)$

$h \quad$ Span

$k_{\text {eff }} \quad$ Effective thermal conductivity

$n \quad$ Wall normal distance

$P \quad$ Local pressure

$P_{0} \quad$ Total pressure

$P_{t} \quad$ Production rate of turbulent kinetic energy

$Q \quad Q$-criterion

Re Reynolds number based on $U_{T E}$ and $C$

$s_{i j} \quad$ Rate of strain tensor

$S \quad$ Blade coordinate based on surface length

$S_{0} \quad$ Total surface length of suction surface

$T$ Temperature

$U \quad$ Velocity magnitude

Subscript

$0 \quad$ Stagnation (total) quantities

in Quantities at the inlet

mid Quantities at the midspan

$T E$ Quantities at the trailing edge

\section{References}

[1] Harrison, S., 1990. "Secondary loss generation in a linear cascade of high-turning turbine blades". Journal of Turbomachinery, 112(4), pp. 618-624. 
[2] MacIsaac, G., Sjolander, S., and Praisner, T., 2012. "Measurements of losses and reynolds stresses in the secondary flow downstream of a low-speed linear turbine cascade". Journal of Turbomachinery, 134(6), p. 061015 .

[3] Denton, J., and Pullan, G., 2012. "A numerical investigation into the sources of endwall loss in axial flow turbines". In ASME Turbo Expo 2012: Turbine Technical Conference and Exposition, American Society of Mechanical Engineers, pp. 1417-1430.

[4] Ong, J., Miller, R. J., and Uchida, S., 2012. "The effect of coolant injection on the endwall flow of a high pressure turbine". Journal of Turbomachinery, $\mathbf{1 3 4 ( 5 ) , \text { p. } 0 5 1 0 0 3 .}$

[5] Reid, K., Denton, J., Pullan, G., Curtis, E., and Longley, J., 2007. "The Interaction of Turbine Inter-Platform Leakage Flow With the Mainstream Flow". Journal of Turbomachinery, 129(2), p. 303.

[6] Steurer, A., Benton, S. I., and Bons, J. P., 2014. "Effect of endwall boundary layer thickness on losses in a lpt cascade with unsteady wakes". In ASME Turbo Expo 2014: Turbine Technical Conference and Exposition, American Society of Mechanical Engineers, pp. V02DT44A037V02DT44A037. 
[7] Pullan, G., 2006. "Secondary flows and loss caused by blade row interaction in a turbine stage". Journal of turbomachinery, 128(3), pp. $484-491$.

[8] Pichler, R., Chen, L., Johnstone, R., and Michelassi, V., 2014. "Compressible direct numerical simulation of low-pressure turbines: Part i-methodology". In Proceedings of the 2014 ASME IGTI Turbo Expo Conference (Düsseldorf, Germany, Citeseer.

[9] de la Blanco, E. R., Hodson, H., Vazquez, R., and Torre, D., 2003. "Influence of the state of the inlet endwall boundary layer on the interaction between pressure surface separation and endwall flows". Proceedings of the Institution of Mechanical Engineers, Part A: Journal of Power and Energy, 217(4), pp. 433-441.

[10] Wu, X., and Jacobs, R., 1999. "Simulation of boundary layer transition induced by periodically passing wakes". Journal of Fluid Mechanics, 398, pp. 109-153.

[11] Xia, H., Tucker, P., Eastwood, S., and Mahak, M., 2012. "The influence of geometry on jet plume development". Progress in Aerospace Sciences, 52, pp. 56-66.

[12] Blanco, E., 2004. "Secondary flows in Low-Pressure Turbines". PhD thesis, University of Cambridge. 
[13] You, D., Wang, M., Moin, P., and Mittal, R., 2007. "Large-eddy simulation analysis of mechanisms for viscous losses in a turbomachinery tip-clearance flow". Journal of Fluid Mechanics, 586, pp. 177-204.

[14] Wissink, J. G., and Rodi, W., 2006. "Direct numerical simulation of flow and heat transfer in a turbine cascade with incoming wakes". Journal of Fluid Mechanics, 569, pp. 209-247.

[15] You, D., Mittal, R., Wang, M., and Moin, P., 2004. "Computational methodology for large-eddy simulation of tip-clearance flows". AIAA journal, $\quad 42(2)$, pp. 271-279.

[16] Rogers, S. E., Kwak, D., and Kiris, C., 1991. "Steady and unsteady solutions of the incompressible navier-stokes equations". AIAA Journal, 29(4), pp. 603-610.

[17] Cui, J., Rao, V. N., and Tucker, P., 2016. "Numerical investigation of contrasting flow physics in different zones of a high-lift low-pressure turbine blade". Journal of Turbomachinery, 138(1), p. 011003.

[18] Opoka, M. M., and Hodson, H. P., 2008. "Experimental Investigation of Unsteady Transition Processes on High-Lift T106A Turbine Blades". Journal of Propulsion and Power, 24(3), May, pp. 424-432.

[19] Denton, J. D., 1993. "The 1993 IGTI Scholar Lecture: Loss Mechanisms in Turbomachines". Journal of Turbomachinery, 115(4), Oct., p. 621. 
[20] Zlatinov, M. B., Tan, C. S., Montgomery, M., Islam, T., and Harris, M., 2012. "Turbine hub and shroud sealing flow loss mechanisms". Journal

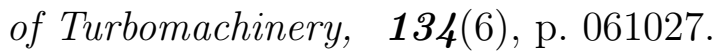

[21] Cui, J., Tucker, P., and Vadlamani, N. R., 2016. "Numerical investigation of secondary flows in a high-lift low pressure turbine". International Journal of Heat and Fluid Flow. in-press.

\section{Appendix A}

A mesh independence study has been performed for a case with a turbulent boundary layer at the inlet. The number of cells for the refined mesh increases to around 85 million. The extra mesh points are used to enhance the mesh resolution in the spanwise and pitchwise directions. Figure 18 shows the averaged grid spacing in wall units for the refined and baseline meshes. The mesh resolution on all the wetted surfaces (pressure surface, suction surface and endwall) are given. The loss coefficient and mean velocity are found to be robust with respect to mesh resolution. See [21] for further details.

In addition to the mean flow quantities, figure 19 shows the pitchwise averaged turbulent kinetic energy profiles. Turbulent kinetic energy is generally more sensitive to the mesh resolution relative to the mean flow quantities. As shown in figure 19, the difference in turbulent kinetic energy between the 40 million and 85 million meshes is imperceptible. 


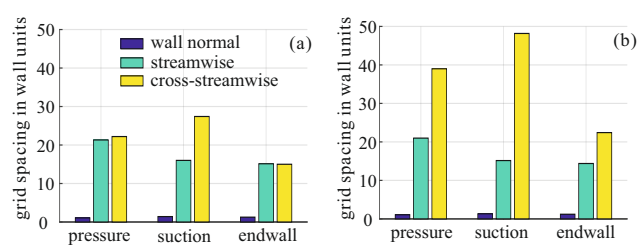

Figure 18: Averaged mesh resolution on the pressure surface, suction surface and endwall: (a) 85 million mesh, (b) 40 million mesh.

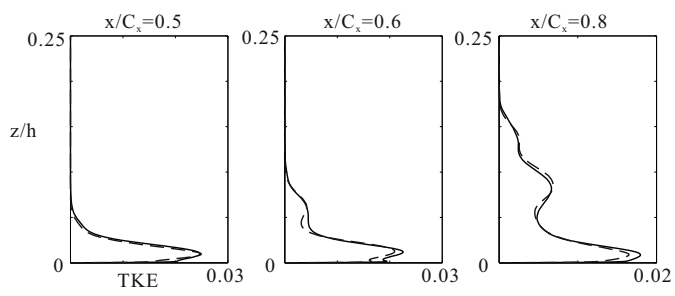

Figure 19: Pitchwise averaged turbulent kinetic energy profiles at (moving from left to right) $x / C_{x}=0.5,0.6$ and $0.8:-40$ million mesh, ----85 million mesh. 